\title{
The Dynamic Topology Changes Model for Unsupervised Image Segmentation
}

\author{
Przemyslaw Lenkiewicz ${ }^{\#, * 1}$, Manuela Pereira ${ }^{\# 2}$, Mário M. Freire ${ }^{\# 3}$, and José Fernandes ${ }^{* 4}$ \\ ${ }^{*}$ Microsoft Portugal \\ Av. Prof. Doutor Aníbal Cavaco Silva, 2744-010 Porto Salvo, Portugal \\ ${ }^{1}$ v-prlenk@microsoft.com \\ ${ }^{4}$ josefer@microsoft.com \\ ${ }^{\#}$ IT-Networks \& Multimedia Group, Department of Computer Science, University of Beira Interior \\ Rua Marquês d'Ávila e Bolama, 6201-001, Covilhã, Portugal \\ mpereira@di.ubi.pt \\ mario@di.ubi.pt
}

\begin{abstract}
Deformable Models are a popular family of image segmentation techniques, which has been gaining significant focus in the last two decades, serving both for real-world applications as well as the base for research work. One of the features that the Deformable Models offer and that is considered a much desired one, is the ability to change their topology during the segmentation process. Using this characteristic it is possible to perform segmentation of objects with discontinuities in their bodies or to detect an undefined number of objects in the scene. In this paper we present our model for handling the topology changes in image segmentation methods based on the Active Volumes solution. The said model is capable of performing the changes in the structure of objects while the segmentation progresses, what makes it efficient and suitable for implementations over powerful execution environment, like multi-core architectures or computer clusters.
\end{abstract}

\section{INTRODUCTION}

The ability to perform topological changes in the structure of objects has been one of the most important features in image segmentation solutions based on Deformable Models [1], [2]. This issue has received a significant amount of research efforts and numerous works have been presented with the aim of classifying and describing different aspects of the topology changing task [2], [3]. In [4] and [5] McInemey and Terzopoulos have considered the incapability of the parametric deformable models for topological transformations without additional mechanisms. They have introduced a model called the T-snake, which was able to dynamically adapt its topology to that of the target object, flow around objects embedded within the target object, and/or automatically merge with other models interactively introduced by the user. A very important solution has been proposed by Casselles [6], who presented a model that was motivated by a curve evolution approach and not an energy minimization one. It allowed automatic changes in the topology when implemented using the level-sets based numerical algorithm [7]. Thereby it allowed the detection of several objects in the scene without previous knowledge of their exact number in the scene and without using special

MMSP'09, October 5-7, 2009, Rio de Janeiro, Brazil.

978-1-4244-4464-9/09/\$25.00 C2009 IEEE. tracking procedures. Their solution has served as the base for numerous following works [8]-[10].

In this paper we present an algorithm called the Dynamic Topology Changes. It serves to automatically create discontinuities in the structure of the Deformable Model in order to detect several objects present in the scene without prior knowledge about their number. Thanks to the ability to estimate its parameters the model does not introduce any need for human supervision over the segmentation process.

The remainder of this paper is organized as follows: Section II describes the Dynamic Topology Changes model and discusses its innovations and advantages. Section III presents image segmentation results that validate the proposed model and present its functionality. Finally, Section IV discusses future work and presents main conclusions.

\section{The Dynamic Topology Changes Model}

The Dynamic Topology Changes model has been developed as a component of the Whole Mesh Deformation model (WMD) [11], which serves for 3-D image segmentation and reconstruction. The WMD requires an efficient and precise scheme for the topology changes due to the following facts: the segmentation process is carried out using a 3dimensional mesh placed over the input data. During this procedure the nodes of the mesh change their positions in a way which will results in placing them over the borders of objects of interests. This approach is similar to the general idea of segmentation methods based on the Deformable Models family and has the most in common with the Topological Active Volumes (TAV) [12] technique, which has served as the foundation for the WMD model. The characteristic of our model that differentiates it from the TAV solution is its ability to take advantage only from the groups of nodes that are initialized in the proximity of objects of interests - the remaining nodes would not be engaged in the segmentation process and at its end they would be discarded. The topology changing mechanism is in that case used to divide the mesh into several disconnected sub-meshes, which would be later classified as either relevant, or irrelevant for the segmentation outcome. Therefore, in the WMD model an 
efficient ability to perform topology changes plays a significant part for the entire segmentation task.

\section{A. The Whole Mesh Deformation Model}

Image segmentation with the WMD model is based on energy function minimization. The said function is defined in such a way to assume its minimal value when the mesh is in the position which segments the shapes of interests present in the input data. In this case, the segmentation is performed by solving an optimization task.

The energy function of the WMD model consists of two types of forces: internal and external ones, which are responsible for preserving the structure of the objects and for applying the features of input images, respectively. In order to calculate the energy for the given model state, the parameter domain $[0,1] \times[0,1] \times[0,1]$ is discretized as a regular mesh defined by the internode spacing and each of the contributing forces described below is calculated using the appropriate algorithm. The internal energy is composed of two forces and it is defined as follows:

$$
E_{\text {int }}(v)=\alpha \sum_{n=1}^{k}\left|\bar{m}-m_{n}\right|+\beta \sum_{n=1}^{k}\left|\arctan \mu_{n}-\frac{\pi}{2}\right|
$$

The two sum expressions represent the mesh continuity and curvature forces. The parameter $\bar{m}$ represents the average distance between all the nodes and $m_{n}$ is the average distance between the node $n$ and its neighbors. The parameter $\mu_{n}$ is the average angle between the node $n$ and its neighbors. As we can see from (1) the continuity force attracts the nodes of the mesh to maintain equal distances between each other, whereas the curvature term attracts the nodes to keep a 90 degrees angle. The external energy is composed of three forces and is defined as follows:

$$
E_{\text {ext }}(v)=\gamma \sum_{n=1}^{k}\left(1-I\left(v_{n}\right)\right)+\delta \sum_{n=1}^{k}\left(1-G\left(v_{n}\right)\right)+\varepsilon \sum_{n=1}^{k} E\left(v_{n}\right)
$$

with $I(v)$ representing the intensity values of the image, $G(v)$ being the Gradient Vector Flow [13] values and $E(v)$ values obtained by the Canny Edge Detector algorithm [14]. The symbols next to the sum expressions $(\alpha, \beta, \gamma, \delta$ and $\varepsilon)$ represent their weights and serve to balance their impact on the whole energy.

The energy optimization is performed using a greedy algorithm approach [15]. During the procedure the following is performed for each node $N$ of the mesh: if the coordinates $\left(\mathrm{x}_{n}, \mathrm{y}_{n}, \mathrm{z}_{n}\right)$ describe the position of the node $N$ at the time $t$, then for time $t+1$ the coordinates of $N$ would be described with $\left(\mathrm{x}_{n+l}, \mathrm{y}_{n+k}, \mathrm{z}_{n}\right)$ where $k, l \in\{-1,0,1\}$ and correspond to the lowest possible value of:

$$
E\left(N_{x+k, y+l, z}\right)=E_{\text {int }(N)}+E_{\text {ext }(N)}
$$

which is the energy of the node $N$ calculated with (1) and (2) at these given coordinates. As it can be seen, the node is moved in its nearest neighborhood in $X$ and $Y$ planes and the position with the lowest energy is chosen as the new position of the node. Those steps are repeated for each node of the mesh until the following rule is satisfied:

$$
\operatorname{sum}_{n} \leq \mu
$$

where $\mu$ is a small value near zero and the $\operatorname{sum}_{n}$ expression is defined as follows:

$$
\operatorname{sum}_{n}=\sum N: N(x, y, z, t) \neq N(x, y, z, t+1)
$$

The given term verifies the number of nodes that have changed their position in the last algorithm iteration. Whenever this number is decreased to zero (or very near to zero) the mesh is assumed to be in its stable position and the segmentation is finished

\section{B. The Dynamic Topology Changes Model}

The WMD model has the ability to change the topology of the mesh during the segmentation process and to create discontinuities in the mesh structure while the mesh deformation progresses. This is performed in contrast to the TAV method, where the task of topology changing is a separate step of the whole segmentation process [12], [16].

The difference between these two ideas is depicted on Figures 1 and 2. The former one shows the approach taken in the TAV solution. It is possible to see that the process is organized in a sequential manner, with each step being performed separately from the remaining ones. The steps of shape optimization are always repeated until the mesh reaches a stable state, namely when the nodes will no longer show a tendency to significantly change their positions. After that the size of the mesh in terms of number of nodes is recalculated in order to provide an even distribution of the nodes in the entire mesh. As a result, the mesh can be deformed out of its optimal shape, which is why the optimization step is performed again. After the abovementioned the step of topology changes is carried out. The mesh is tested for nodes placed in wrong locations, like the external nodes placed far from any surface or internal nodes placed on a background area. Those nodes, when detected, are subjected to one of the following

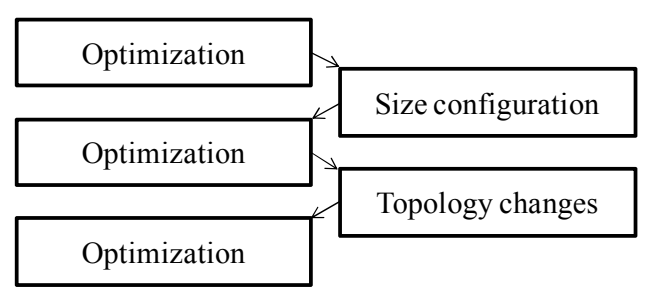

Fig. 1. Organization of the processes composing the segmentation procedure in the case of TAV solution.

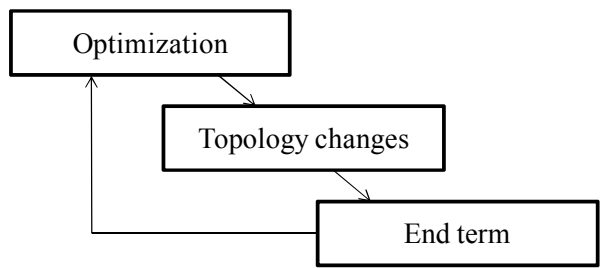

Fig. 2. Organization of the processes composing the segmentation procedure in the case of WMD model. 
procedures: the node removal or the link removal. The former assumes that the wrongly located node should be discarded completely from the mesh structure; the latter assumes that only the links interconnecting the given node with its neighbors should be broken until the structure of the mesh will not show any more errors. Finally, the shape optimization step is carried out again to ensure that the mesh assumes the position with the minimal energy value.

As it can be seen, the composition of the process is modular. The respective parts are performed one by one and any step along the sequence requires for the predecessor to finalize its actions. This causes the segmentation process to be very badly suited for tasks like workload parallelization. Also, the progression of the model towards the desired result is not stable and during a major part of the segmentation process the state of the model represents data that is not useful.

Approach used as the base for the Dynamic Topology Changes model is presented on Fig. 2. It is constructed using a different assumption, namely to join the topology changes feature together with the optimization of mesh and carry them out along the whole segmentation process. Whenever a need for mesh reconfiguration would be encountered during the shape optimization, the model should react in desired way and start creating discontinuities in the mesh structure. On the other hand, the said topology changes feature should be constrained at all times by the mesh energy function, which would guarantee that it will not cause it to progress out of its stable state. This twofold dependency between two processes would assure the correct behavior of the mesh and a stable progression to its optimal shape.

\section{The Procedure of Topology Changing}

As shown on the Fig. 2, the topology changing and the shape optimization steps are constrained into one loop, which is repeatedly executed until the segmentation end term has been met. During the shape optimization step each node of the mesh can change its position due to the energy minimization process and as a result the lengths of links connecting it with its neighbors are also altered. What follows is the initialization of the topology changing mechanism in order to verify if any discontinuities in the mesh should be created. This is done with the following steps: the lengths of the links between the given node and its neighborhood are checked with the following term:

$$
\left|\operatorname{link}\left(N_{n}, N_{c}\right)\right| \leq \frac{G_{x}+G_{y}}{2} \times \text { flex }
$$

where $G_{x}$ and $G_{y}$ represent the average distance between mesh nodes in $x$ and $y$ directions and flex is the flexibility parameter, which is defined upon segmentation execution and will be discuss later in section II D. Whenever a certain link fails to satisfy (6) it is marked as broken. We can safely assume that this process is carried out only when desired because the mesh is defined to have a rigid and stable structure and as a result a majority of the links of the mesh extend their lengths only by small values during the whole process. When above described situation of a link breaking occurs, we can assume with high probability that a given node has been attracted to a border of an object present in the scene of interests and thus the connection breaking should be called.

The broken link will no longer be considered when calculating the continuity term of the energy function in the next iteration. As a result, the node will show a behavior as if this link did not exist at all and it will progress away from its current location more freely. This will usually cause extending and possibly breaking the links of its neighbors, as shown on Fig. 3. This sort of chain reaction is much desired as it will trigger the movement of nodes and breaking of connections in a small neighborhood. This will in turn lead to a successful detection of the entire discontinuity in the mesh structure. Such process will be stopped at the right locations, namely where the nodes of the mesh encounter the edges of objects of interest. This is guaranteed by defining the energy of the edge detector to a very significant value, which will always stop the progression of nodes.

\section{Estimation of the flexibility parameter}

The Dynamic Topology Changes mechanism requires the correct value of the flexibility parameter to be defined upon algorithm execution in order to perform successful segmentation. If the said parameter would be defined with a value that is too high for the specific environment, the mesh would not be disconnected in the areas in which it should be and as a result several objects present in the scene would be detected as one. On the other hand, if the flexibility parameter would be defined too low, the mesh would detect discontinuities in locations where they actually do not exist. Manual selection of this parameter for each segmentation task is much undesired. To eliminate the need for this we have developed a function that calculates the correct value of the flexibility parameter according to the current execution environment.

To do this we have performed a number of segmentation experiments using a set of artificial images as the input. Those have been constructed with the aim of representing several different scenarios concerning the features, which in our opinion would have an impact on the required value of the flexibility parameter. Those are namely:

- the minimum distance between the objects in the scene

- the size of the smallest discontinuity in the scene

- the width of the largest object in the scene

After performing the first round of experiments we have discovered that the distances enumerated above should be measured in a slightly different manner, namely not as the

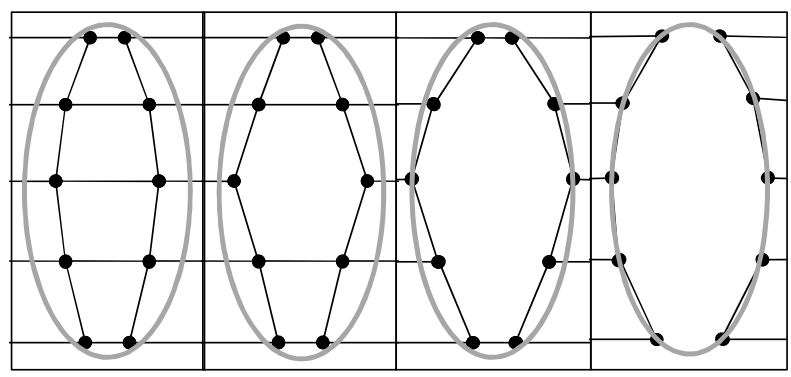

Fig. 3. Example of a chain reaction during discontinuity detection in the structure of an object. 
actual distance in a two-dimensional plane, but rather as two separate distances in the $\mathrm{x}$ and $\mathrm{y}$ planes individually. Then, the smaller value should be considered as relevant. The style of this measurement is depicted on Fig. 4. The explanation for this fact lies in the way in which the mesh is constructed. It is initialized with the links forming a regular, cubic structure and during the segmentation process the mesh tends to maintain this arrangement. As a result majority of the links would still be located in a parallel manner either to the $\mathrm{x}$ or $\mathrm{y}$ axis when they will be subjected to testing with the flexibility term and thus those distances should also be measured in a manner parallel to the $\mathrm{x}$ and $\mathrm{y}$ planes.

The experiments that we have performed were carried out according to the following plan:

- A series of artificial images presented in Fig. 5 was used.

- For each of the images we have measured the three values described above.

- For each image we have performed a series of segmentation experiments using different values of the flexibility parameter and of the mesh grid size. The value that offered the best segmentation result was marked as the optimal one for the given scenario.

Using a total number of 16 images with 4 different grid sizes and about 5-6 values of the flexibility parameter, we have performed 352 segmentation experiments. During these experiments it was observed that:

- A strong dependency can be noted between flexibility parameter and the initial size of the grid. The optimal value of the parameter has been kept in the range of:

$$
\text { flex }(\text { grid }) \in(1.8 \times \text { grid } ; 2.1 \times \text { grid })
$$

where flex stands for the value of the flexibility parameter and grid is the average initial size of the mesh in $x$ and $y$ directions. This is a very expected behavior, as both the initial grid size and the flexibility parameter describe in a straightforward way the same feature of the mesh during the segmentation process, namely the lengths of the links.

- The exact optimal value of the flexibility parameter depends on the minimum distance between the objects in the image or the size of the smallest discontinuity whichever was smaller. This dependency is depicted on Fig. 6. As it can be seen, the correlation of the both

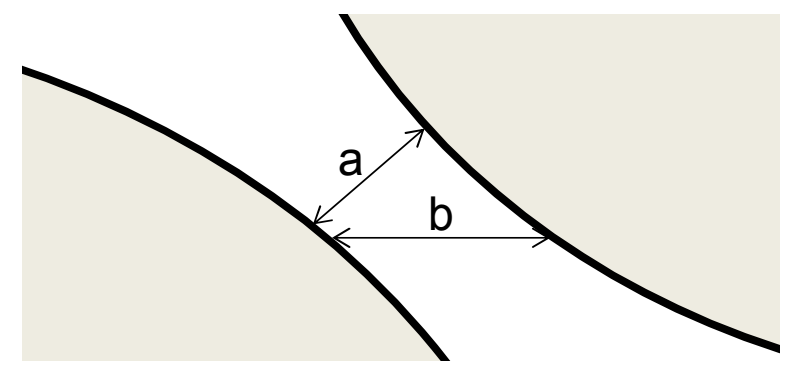

Fig. 4. Illustration of the way in which the measurements of image features in our experiments are taken. The value $a$ is the actual distance between the two objects in the image, the value $b$ is the distance measured only in the $X$ plane. The latter is considered as relevant in our experiments.

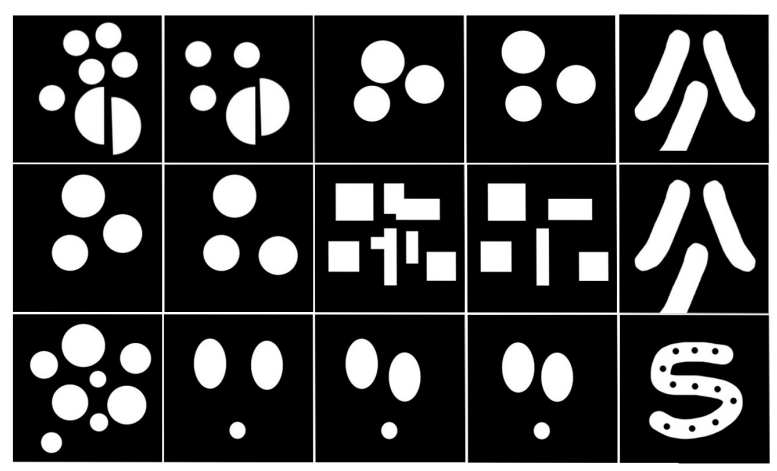

Fig. 5. A set of artificial images used to perform our experiments.

values is high and the value of the optimal flexibility parameter can be expressed as follows:

$$
\text { flex }(\text { dist })=\left\{\begin{aligned}
\min _{d}, & \text { dist }<\min _{d} \\
\text { dist, } & \text { dist } \in\left[\min _{d}, \max _{d}\right] \\
\max _{d}, & \text { dist }>\max _{d}
\end{aligned}\right.
$$

where $\min _{d}$ and $\max _{d}$ stand for the minimum and maximum values from the optimal range of the flexibility parameter according to (7) and the dist described the smallest distance between the objects in the scene.

- The width of the objects themselves does not show to have any impact on the optimal flexibility parameter value.

- Finally, the algorithm shows to allow some margin of error, i.e. the segmentation outcome has not been noticeably different with the flexibility parameter set to $\pm 15 \%$ of its optimal value.

\section{VALIDATION OF THE PROPOSED METHOD}

To assess the efficiency of our topology changing method we have performed a series of test, each presenting different type of input data. The first group was based on artificial images, not used before as the training set for the construction of the function calculating the dynamic flexibility parameter. The second group consisted of real, 2-dimensional medical images. The third experiment included a 3-dimensional volume representing a CT scan of a human brain and composed of a series of 12 slice images.

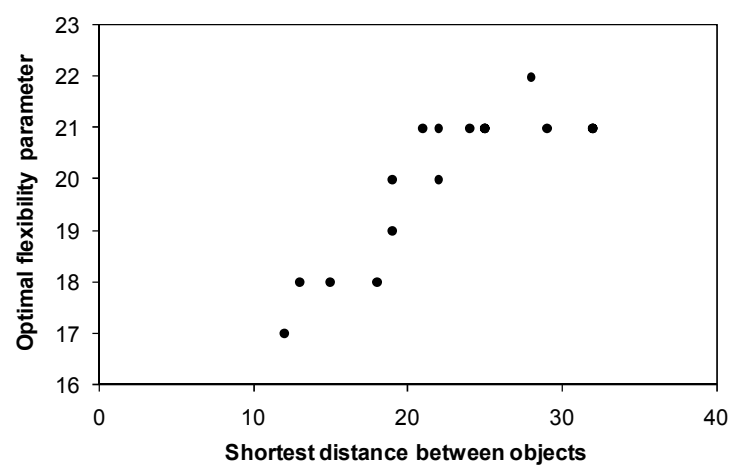

Fig. 6. Dependency between the optimal value of the flexibility parameter and the shortest distance between objects in the scene. 
At the beginning of the segmentation process the input images have been subjected to an automatic measurement function that has analyzed them for the size of discontinuities in directions parallel to $\mathrm{x}$ and $\mathrm{y}$ planes. Images representing the edge detector function have been used for this purpose and since the measurement had to be taken directly in $\mathrm{x}$ and $\mathrm{y}$ directions, this process was simple and not time consuming. As the second step, the same experiments have been performed with the flexibility parameter manually chosen to an optimal value. In order to avoid any influence on this decision, this was done without the knowledge about the value chosen for this particular scenario by the automatic function. In all the cases the difference between values chosen in manual and automatic manner has been kept on a very small level of $10-15 \%$ of the value, which corresponded to $1-2$ pixels. As said before, such a small difference was difficult to notice in the results and small enough to be ignored.

Examples of the results delivered by the segmentation algorithm are presented on Fig. 7. The medical images used for this experiment have been subjected to a supervised contrast enhancement in order to remove the unwanted parts. As it can be seen, the topology changes are performed successfully; the discontinuities in the structure of objects are detected and the mesh is divided into separate parts. Also, the unwanted parts of the mesh are detected successfully and discarded from the segmentation results. This can be seen on Fig. 8, where the consecutive steps of the segmentation process are presented.

\section{CONCLUSION}

In this paper we have presented the Dynamic Topology Changes model, which is a component of the Whole Mesh Deformation model for image segmentation. The process of topology changing is integrated in the shape optimization task, which makes the whole segmentation a stable process, with a straight progression of the model towards its optimal state. Thanks to the ability to analyze the scene of interests with a simple and fast function, the model is able to provide a

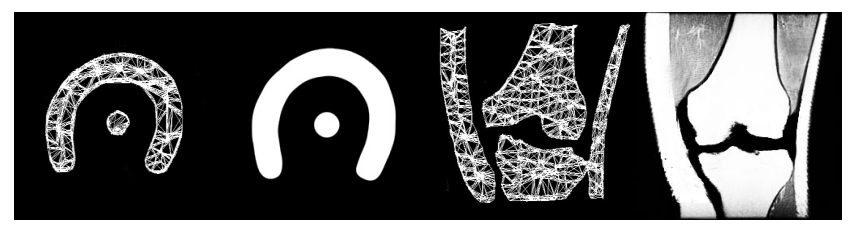

Fig. 7. Examples of results obtained with our segmentation algorithm. The results and their sources do not represent pixel-to-pixel correspondence.
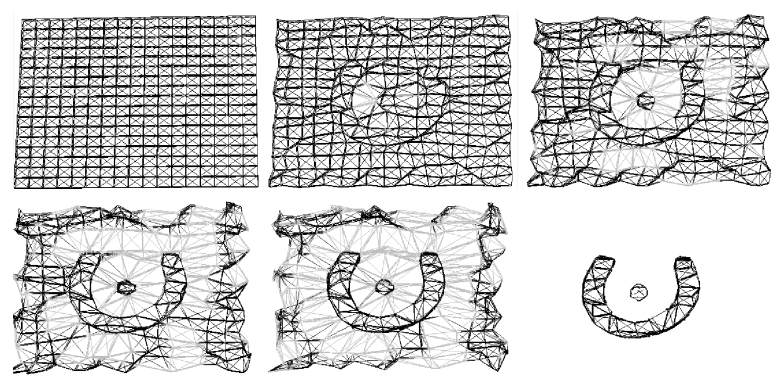

Fig. 8. Consecutive steps of segmentation of an artificial shape used in the method validation process. successful detection of image features without the need to provide any additional parameters from the side of the user.

Our interests for the future work include extending the Dynamic Topology Changes model with the ability to change the value of the flexibility parameter during the segmentation procedure and to calculate the said parameter with the respect to a certain regions. These features would make the model much more robust in scenes with uneven distribution of the objects and high variety of the shapes.

\section{ACKNOWLEDGMENT}

This work has been partially funded by Microsoft Portugal and by the University of Beira Interior through the HIPERCAMBIO Project, and by the Institute of Telecommunications, Portugal.

\section{REFERENCES}

[1] M. Kass, A. Witkin, and D. Terzopoulos, "Snakes: Active contour models," International Journal of Computer Vision, vol. 1, pp. 321-331, 1988.

[2] T. McInerney and D. Terzopoulos, "Deformable models in medical image analysis: a survey," Medical Image Analysis, vol. 1, pp. 91-108, 1996.

[3] H. Delingette and J. Montagnat, "Shape and topology constraints on parametric active contours," Comput. Vis. Image Underst., vol. 83, pp. 140-171, 2001.

[4] T. McInerney and D. Terzopoulos, "Topologically adaptable snakes," in Proc. Fifth International Conference on Computer Vision, 1995, pp. 840-845.

[5] T. McInemey and D. Terzopoulos, "Topology adaptive deformable surfaces for medical image volume segmentation," IEEE Transactions on Medical Imaging, vol. 18, pp. 840-850, 1999.

[6] V. Caselles, "Geometric models for active contours," in Proc. International Conference on Image Processing, 1995, vol.3, pp. 9-12.

[7] S. Osher and J. A. Sethian, "Fronts propagating with curvaturedependent speed: algorithms based on Hamilton-Jacobi formulations," J. Comput. Phys., vol. 79, pp. 12-49, 1988.

[8] K. Fritscher and R. Schubert, "3D image segmentation by using statistical deformation models and level sets," International Journal of Computer Assisted Radiology and Surgery, vol. 1, pp. 123-135, 2006.

[9] M. E. Leventon, W. E. L. Grimson, and O. Faugeras, "Statistical shape influence in geodesic active contours," in Proc. IEEE Conference on Computer Vision and Pattern Recognition, 2000, vol.1, pp. 316-323.

[10] R. Malladi, J. A. Sethian, and B. C. Vemuri, "Shape modeling with front propagation: a level set approach", IEEE Transactions on Pattern Analysis and Machine Intelligence, vol. 17, pp. 158-175, 1995.

[11] P. Lenkiewicz, M. Pereira, M. Freire, and J. Fernandes, "A New 3D Image Segmentation Method for Parallel Architectures," in Proc. IEEE International Conference on Multimedia and Expo, New York City, USA, 2009.

[12] N. Barreira, M. G. Penedo, C. Mariño, and F. M. Ansia, "Topological Active Volumes," in Computer Analysis of Images and Patterns, 2003, pp. 337-344.

[13] X. Chenyang and J. L. Prince, "Gradient vector flow: a new external force for snakes," in Proc. IEEE Computer Society Conference on Computer Vision and Pattern Recognition, 1997, pp. 66-71.

[14] J. F. Canny, "A computational approach to edge detection," in Readings in computer vision: issues, problems, principles, and paradigms: Morgan Kaufmann Publishers Inc., 1987, pp. 184-203.

[15] D. Williams and M. Shah, "A Fast algorithm for active contours and curvature estimation," CVGIP: Image Understanding, vol. 55, pp. 14$26,1992$.

[16] N. Barreira, M. G. Penedo, C. Alonso, and J. Rouco, "Handling Topological Changes in the Topological Active Volumes Model," in Proc. of the 5th international conference on Image Analysis and Recognition. vol. 5112/2008, Póvoa de Varzim, Portugal: SpringerVerlag, 2008, pp. 122-131. 